\title{
Análise de Sobrevida Global e Fatores de Risco para Óbito em 509 Pacientes com Lúpus Eritematoso Sistêmico (LES) ${ }^{*}$
}

\section{Analysis of Global Survival and Risk Factors for Death in 509 Systemic Lupus Erythematosus (SLE) Patients}

\author{
Simone Appenzeller ${ }^{(1)}$ e Lilian Tereza Lavras Costallat ${ }^{(2)}$
}

\section{RESUMO}

Estudos epidemiológicos com análise multivariada de fatores prognósticos em pacientes com lúpus eritematoso sistêmico (LES) são fundamentais para avaliar a sobrevida e demonstrar estratégias de diagnóstico e tratamento. Objetivo: analisar os fatores de risco para a mortalidade em uma coorte de 509 pacientes com LES. Métodos: os pacientes foram acompanhados em um hospital terciário, por um período de 27 anos (1974-2001), de acordo com um protocolo previamente padronizado. Inicialmente, foi realizada a análise de sobrevida global até o óbito dos 509 pacientes e, posteriormente, foi verificada a diferença entre as probabilidades de sobrevida global para pacientes com início da doença antes dos 16 anos e após os 16 anos. Resultados: a taxa de sobrevida para os 509 pacientes com LES após 1, 5, 10 e 20 anos foram respectivamente de $96 \%, 88 \%, 80 \%$ e $75 \%$. Os fatores prognósticos para o óbito dos pacientes com LES foram a ausência de artrite ao diagnóstico $(\mathrm{p}=0,007 ; \mathrm{OR}=0,45 ; 95 \% \mathrm{IC}=0,25-0,8)$, a ausência do uso de antimaláricos $(\mathrm{p}=0,0001 ; \mathrm{OR}=0,3 ; 95 \% \mathrm{IC}=0,2-0,5)$, a presença de hipertensão arterial $(\mathrm{p}=0,002 ; \mathrm{OR}=2,2 ; 95 \% \mathrm{IC}=1,1$ $4,5)$, a presença de infecções $(p=0,003 ; \mathrm{OR}=2,3 ; 95 \% \mathrm{IC}=1,3$ $4,1)$ e a idade ao diagnóstico menor que 16 anos $(\mathrm{p}=0,004$; $\mathrm{OR}=2,6 ; 95 \% \mathrm{IC}=1,4-5,0)$. Conclusões: este trabalho mostra uma excelente taxa de sobrevida nesta casuística de 509 pacientes com LES, observando-se proporção de pacientes que sobreviveram após 1, 5, 10 e 20 anos de seguimento, respectivamente, de $96 \%, 88 \%$, $80 \%$ e $75 \%$, comparável às maiores séries que tratam de sobrevida.

Palavras-chave: lúpus eritematoso sistêmico (LES), mortalidade, sobrevida, fatores de risco.

\begin{abstract}
Epidemiological studies using multivariate analysis of risk factors in Systemic Lupus Erythematosus (SLE) patients are essential to determinate survival and to demonstrate diagnosis and treatment strategies. Objective: To analyse the risk factors of mortality in 509 SLE patients who were followed over a 27-year period (from 1974 to 2001) according to a standard protocol in a single centre. The first analysis consisted of the survival time from the date of diagnosis of SLE until the last contact or death. The second analysis consisted of the survival time from the date of diagnosis of SLE until the last contact or death of patients whose disease started before 16 years old compared to patients whose disease started after 16 years old. Methods: The percentage of survival after 1, 5, 10 and 20 years were $96 \%, 88 \%, 80 \%$ and $75 \%$. The risk factors for death in SLE patients were the absence of arthritis at diagnosis $(p=0.007 ;$ OR $=0.45 ; 95 \%$ IC $=0.25-0.8)$, the absence of chloroquine sulphate prescription $(p=0.0001 ;$ OR $=0.3$; $95 \% C I=0.2-0.5)$, the presence of hypertension ( $p=0.002$; $O R=2.2 ; 95 \% C I=1.1-4.5)$, the presence of infections $(p=0.003 ; O R=2.3 ; 95 \% C I=1.3-4.1)$ and less than 16 years old at diagnosis $(p=0.004 ; O R=2.6 ; 95 \% C I=1.4-5.0)$. Conclusions: This paper presents an excellent survival in this cohort of 509 SLE patients. The percentage of survival after 1, 5, 10 and 20 years, respectively 96\%, 88\%, 80\% and 75\%, was similar to other studies.
\end{abstract}

Keywords: Systemic Lupus Erythematosus, mortality, survival, risk factors.

\footnotetext{
* Departamento de clínica médica da Faculdade de Ciências Médicas da Universidade Estadual de Campinas (FCM/Unicamp). Trabalho realizado com bolsa de iniciação científica da Fapesp e com auxílio parcial do Fundo de Auxílio à Pesquisa e Ensino em Reumatologia da Sociedade Brasileira de Reumatologia. Laureado com o Prêmio Jovem Cientista da Década do Osso e da Articulação no XXIV Congresso de Reumatologia. Recebido em 27/03/2003. Aprovado, após revisão, em 15/01/2004.

1. Pós-graduanda do departamento de clínica médica da Unicamp.

2. Professora titular da disciplina de Reumatologia da Unicamp.

Endereço para correspondência: Dra. Lilian T.L. Costallat. Disciplina de Reumatologia. Departamento de clínica médica da Faculdade de Ciências Médicas da Universidade Estadual de Campinas (FCM/Unicamp), CEP 13081-970, Barão Geraldo, Campinas, São Paulo, SP.
} 


\section{INTRODUÇÃO}

O lúpus eritematoso sistêmico (LES) é uma doença crônica cuja fisiopatologia envolve mecanismos auto-imunes e inflamatórios, com evolução apresentando períodos de atividade ou remissão e manifestações multissistêmicas ${ }^{(1)}$.

O prognóstico dos pacientes com LES tem melhorado muito nos últimos 40 anos. Em 1954, 78\% dos pacientes sobreviviam ao primeiro ano da doença e apenas $52 \%$ chegavam ao quarto ano ${ }^{(2,3)}$. Em 1982, as porcentagens de sobrevida de 1 ano, 4 anos e 10 anos passaram a ser de 95\%, $88 \%$ e $76 \%$, respectivamente ${ }^{(4)}$. Publicações recentes têm mostrado taxas de sobrevida de $95 \%$ aos 5 anos, $90 \%$ aos 10 anos e de $79 \%$ a $83 \%$ aos 15 anos $^{(5,6,7,8)}$.

O aumento das taxas de sobrevida tem sido atribuído a vários fatores, tais como: diagnósticos precoces, melhores opções terapêuticas e controle das condições mórbidas associadas $^{(9)}$.

As causas de morte no LES podem estar associadas à atividade da doença e às complicações do seu tratamento, além de outras causas não relacionadas diretamente com a patologia ${ }^{(2,9)}$.

Pior prognóstico é reconhecidamente associado ao acometimento renal ${ }^{(4,10,11)}$ e do sistema nervoso central (SNC). No que se refere às causas de óbito relacionadas com o tratamento do LES, a infecção, freqüentemente associada à imunossupressão, tem sido implicada como a principal causa $^{(7,9,12)}$. Com a melhora na sobrevida dos pacientes com LES tem-se observado aumento na incidência de doenças cardiovasculares, cuja causa está relacionada diretamente com a atividade da doença e com o uso prolongado de corticosteróides, além de outros fatores de riscos como hipertensão arterial, diabetes, sedentarismo e obesidade.

A análise da sobrevida em pacientes lúpicos é de fundamental importância, pois pode traçar estratégias de monitoramento e avaliar a eficácia da terapêutica.

Portanto, o objetivo deste estudo foi avaliar os fatores relacionados com a sobrevida em 509 pacientes lúpicos seguidos em um hospital universitário de complexidade terciária.

\section{PACIENTES E MÉTODOS}

\section{PACIENTES}

Foram estudados todos os pacientes internados e/ou acompanhados no serviço de Reumatologia do Hospital das Clínicas da Unicamp, com diagnóstico de LES definido segundo os critérios do Colégio Americano de Reumatologia (ACR) $)^{(13)}$ de 1974 até 2001.

\section{DELINEAMENTO DO ESTUDO}

O estudo foi do tipo coorte, sendo que os pacientes foram acompanhados desde a data do diagnóstico firmado por médico, com base nos critérios já mencionados, até a data do óbito ou a data da última consulta no serviço de Reumatologia da Faculdade de Ciências Médicas da Unicamp. A data final de seguimento, para este trabalho, foi 31 de julho de 2001.

\section{INSTRUMENTO DE COLETA}

Todos os prontuários da disciplina de Reumatologia foram transcritos em protocolos previamente padronizados. Neste protocolo foram anotados dados completos de história clínica, exames subsidiários, tratamento e evolução e, posteriormente, passados para o computador no programa Epiinfo 6.0. As informações dos pacientes, cuja doença foi diagnosticada em outro local, foram coletadas através de contato telefônico ou por carta.

As causas de óbito foram definidas a partir das informações médicas do registro hospitalar ou usando a causa relacionada nos atestados de óbito (óbito fora do serviço), fornecido pelos parentes ao serviço hospitalar.

As variáveis e as categorias analisadas foram as seguintes: sexo; raça; idade: idade de início do diagnóstico da doença (foram analisadas como um grupo único e, posteriormente, em duas faixas etárias: início antes dos 16 anos e após os 16 anos); tempo de doença (do início do diagnóstico até o dia 31 de julho de 2001 ou até a data do óbito); tempo de seguimento (do início do acompanhamento neste serviço até o dia 31 de julho de 2001 ou até a data do óbito).

As variáveis clínicas, laboratoriais e o uso de imunossupressores que se seguem foram analisados quando do diagnóstico do LES e durante o seguimento dos pacientes. Além dos critérios clínicos do LES definidos pelo ACR, foram pesquisados: adinamia; emagrecimento $(>4 \mathrm{~kg})$; febre $\left(\geq 37,8^{\circ} \mathrm{C}\right)$; necrose asséptica; deformidades articulares (geralmente redutíveis); alopecia; hipertensão arterial (HAS): pressão sistólica maior que $130 \mathrm{mmHg}$ e/ou pressão diastólica maior que $90 \mathrm{mmHg}$ ); manifestações pulmonares como hipertensão pulmonar, pneumonite e hemorragia pulmonar; manifestações cardíacas como miocardite, endocardite própria do SLE e infarto do miocárdio e miopatia. Considerou-se também o envolvimento intestinal, hepático, e do sistema retículo-endotelial, bem como a presença de tromboembolismo pulmonar, fenômeno de Raynaud e alterações oculares. Informações pertinentes à concomitância de outras doença auto-imunes (DAI) ou história familiar de DAI, foram consideradas, além daquelas referentes à gestações, partos, abortos e natimortos. 
A terapêutica empregada durante a evolução da doença foi anotada.

Todos os exames laboratoriais e auto-anticorpos foram realizados seguindo técnicas de rotina utilizadas no Laboratório de Patologia Clínica e no Laboratório de Investigação em Alergia e Imunologia da Unicamp. As alterações hematológicas, quando induzidas por drogas ou infecções, foram excluídas. Considerou-se, além dos critérios laboratoriais para o LES, anticorpo anticardiolipina (por método imunoenzimático) e anticoagulante lúpico (por TTPA e Russel).

\section{ANÁLISE ESTATÍSTICA}

Primeiramente, foi feita a análise descritiva das características dos pacientes. Para verificar associações entre as variáveis categóricas foi utilizado o teste qui-quadrado. Quando os valores esperados foram menores que 5 , utilizou-se o teste exato de Fischer, sendo considerado significativa a associação quando o valor do $\mathrm{p}$ era menor ou igual a 0,05 .

Em seguida, realizou-se a análise de sobrevida até o óbito (global). Para a análise da sobrevida até o óbito, os pacientes foram acompanhados desde a data do diagnóstico até a data de seu óbito. Foram excluídos os pacientes que não compareceram ao serviço há mais de um ano, aqueles que estavam vivos na data do término do estudo, bem como os pacientes que foram a óbito por causas não relacionadas direta ou indiretamente com a doença de base.

A análise de sobrevida foi, inicialmente, univariada, através do estimador produto limite de Kaplan Meier ${ }^{(14)}$, para se avaliar dentre as variáveis de estudo quais foram os fatores prognósticos mais significantes para o óbito. A comparação entre as curvas de sobrevivência acumulada foi feita pelo teste estatístico de log-rank. E em alguns casos foi utilizado o teste de Wilcoxon (Breslow), quando a propriedade de riscos proporcionais não foi verificada.

A seguir foi feita a análise multivariada mediante o modelo de riscos proporcionais de $\mathrm{Cox}^{(15)}$ para se verificar a atuação conjunta de todos os fatores prognósticos. Neste trabalho elaboraram-se primeiramente modelos de Cox univariados para cada uma das variáveis de estudo. $\mathrm{Na}$ análise multivariada, iniciou-se o processo de modelagem pela variável mais significante, do ponto de vista estatístico, no modelo univariado de Cox; a seguir foram acrescentadas, no modelo, as outras variáveis, uma a uma, seguindo a ordem decrescente de significância estatística. A nova variável era mantida no modelo multivariado caso fosse considerada estatisticamente significante. Também era levada em conta a importância clínica da variável. Uma variável era considerada de confusão quando alterava o coeficiente de regressão de outra variável já existente no modelo. Após a introdução de todas as variáveis no modelo, testou-se o efeito de interação dentre aquelas em que se verificou o efeito de confusão.

Como resultado final da análise multivariada, foram estimadas as razões das funções de risco (RR) para cada uma das variáveis constantes no modelo final. A estimativa foi feita por ponto e por intervalo de confiança de $95 \%$.

\section{RESULTADOS}

\section{DESCRIÇÃO DOS PACIENTES}

A casuística deste estudo compreendeu um total de 509 pacientes, com mediana de idade ao diagnóstico de 25 anos (mínimo de 5 e máximo de 62 anos; desvio padrão $[D P]=10,3$ anos). $O$ tempo de doença variou de um mês a 28 anos, em média de 4,6 anos ( $\mathrm{DP}=4,5$ anos). O tempo médio de seguimento foi de 3,1 anos ( $\mathrm{DP}=3,5$ anos).

As características demográficas e as manifestações clínicas destes pacientes encontram-se, respectivamente, na Tabela 1 e Figura 1.

TABELA 1

LES: CARACTERÍSTICAS DEMOGRÁFICAS EM 509 PACIENTES COM LES

\begin{tabular}{lcc}
\hline $\begin{array}{l}\text { Características } \\
\text { demográficas }\end{array}$ & $\begin{array}{c}\text { Coorte de } 509 \\
\text { pacientes }\end{array}$ & $\begin{array}{c}\text { Pacientes que } \\
\text { foram a óbito (58) }\end{array}$ \\
\hline $\begin{array}{l}\text { Sexo } \\
\text { feminino/masculino }\end{array}$ & $\begin{array}{c}471(92,53 \%) / \\
38(7,46)\end{array}$ & $\begin{array}{c}51(10,2 \%) / \\
7(1,4 \%)\end{array}$ \\
$\begin{array}{l}\text { Relação sexo } \\
\text { feminino/masculino }\end{array}$ & $12: 1$ & $7,3: 1$ \\
$\begin{array}{l}\text { Caucasóides/ } \\
\text { não caucasóides }\end{array}$ & $392(77,01 \%) /$ & $43(8,5 \%)$ \\
$\begin{array}{l}\text { Idade de início do } \\
\text { LES (anos } \pm \text { EP) }\end{array}$ & $26,20 \pm 10,12$ & $15(3,0 \%)$ \\
$\begin{array}{l}\text { Idade ao diagnóstico } \\
\text { (anos } \pm \text { EP) }\end{array}$ & $26,80 \pm 10,33$ & $21,10 \pm 9,20$ \\
\hline $\begin{array}{l}\text { Tempo de diagnóstico } \\
\text { (anos } \pm E P \text { ) }\end{array}$ & $4,67 \pm 4,54$ & $3,27 \pm 2,1$ \\
$\begin{array}{l}\text { Tempo de seguimento } \\
\text { (anos } \pm \text { EP) }\end{array}$ & $3,13 \pm 3,52$ & $3,3 \pm 2,12$ \\
\hline EP: erro padrão & &
\end{tabular}




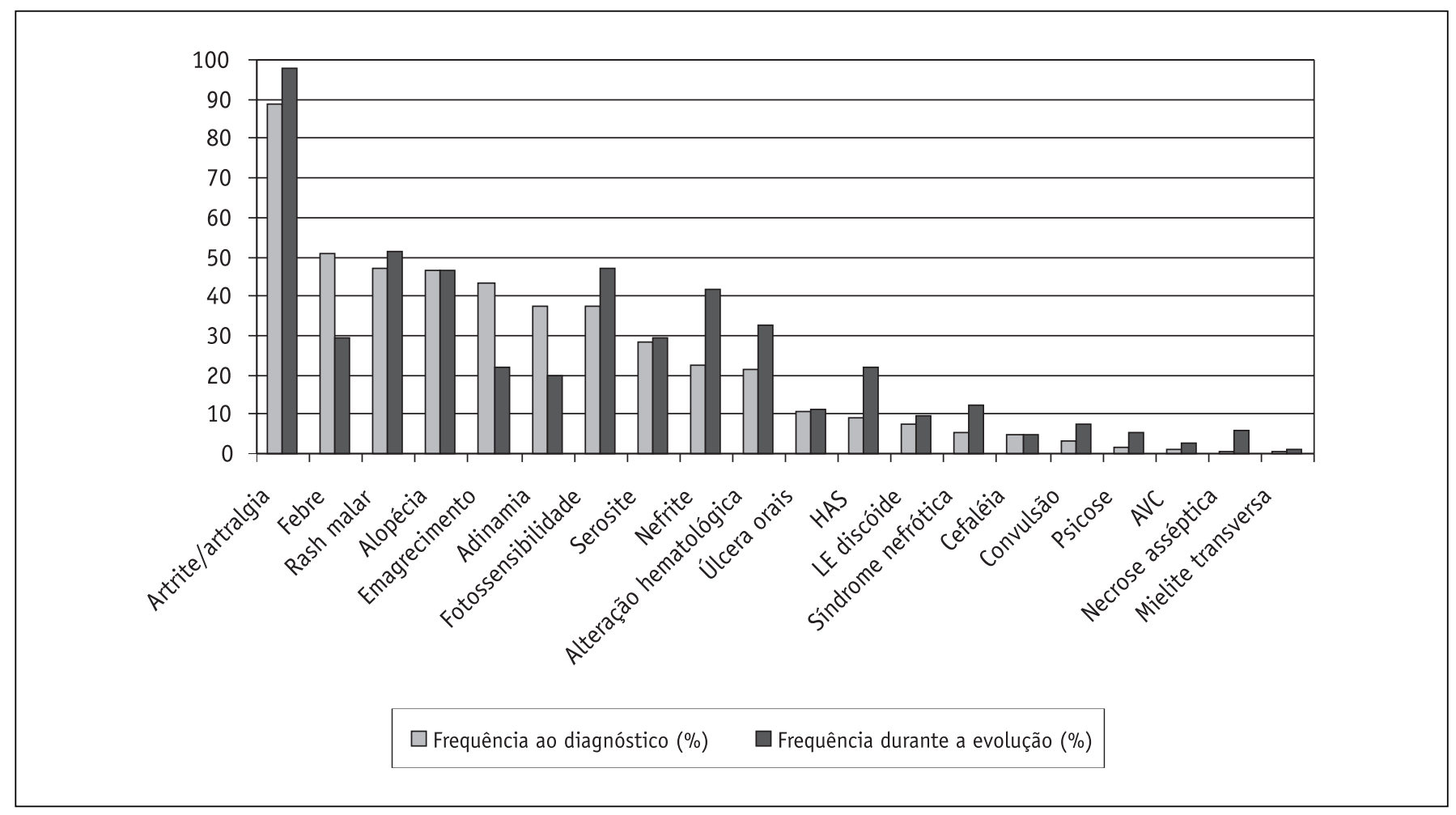

FiguRA 1- LES: Manifestações clínicas ao diagnóstico e na evolução em 509 pacientes.

CAUSAS DE óbITO E FATOREs ASSOciados

Ocorreram, no total, 58 óbitos $(11,4 \%)$ durante o seguimento desta coorte. Proporcionalmente, ocorreram mais óbitos no sexo feminino que no masculino (respectivamente $87,9 \%$ e $12,0 \%$ ), entretanto, esta diferença não foi estatisticamente significante.

As causas de óbito foram: sepse (43,1\%), insuficiência respiratória $(24,1 \%)$, acidente vascular cerebral $(6,9 \%)$, falência cardiovascular $(5,2 \%)$ tamponamento cardíaco $(1,7 \%)$, meningococcemia $(1,7 \%)$, neoplasia (1,7\%) e suicídio (1,7\%). Em 13,8\% dos pacientes, as causas de óbito não puderam ser determinadas.

As variáveis independentes associadas ao óbito foram ao diagnóstico da doença: a ausência de sintomas gerais $(p=0,026)$ a presença de nefrite $(p=0,028)$ a presença de HAS $(p=0,005)$, a presença de acidente vascular cerebral $(p=0,012)$ e a presença de plaquetopenia $(p=0,046)$. Durante a evolução da doença, as variáveis associadas ao óbito foram a ausência de uso de antimalárico $(p=0,002)$, a presença de infecções $(p=0,001)$, o uso de pulso de corticóide ( $\mathrm{p}=0,001)$ e o uso de pulso de ciclofosfamida $(p=0,021)$.

\section{ANÁlISE dA SOBREVIDA ATÉ O ÓBITO (SOBREVIDA GLOBAL)}

Os valores estimados das proporções de sobrevida global para os pacientes com LES, segundo o método de Kaplan Meier, foram de $96 \%$ ao $1 .^{\circ}$ ano, $93 \%$ aos 2 anos, $88 \%$ aos 5 anos, $80 \%$ aos 10 anos, $75 \%$ aos 20 anos (Figura 2).

\section{A PROBABILIDADE DE SOBREVIDA ACUMULADA SEGUNDO AS VARIÁVEIS ESTUDADAS}

Ao comparar-se as curvas de probabilidade de sobrevida acumulada entre os sexos, observou-se que a sobrevida dos pacientes do sexo masculino foi sempre inferior quando comparada ao sexo feminino, embora não tenha sido detectada diferença estatisticamente significante entre os dois grupos $(p=0,33)$. Após 5 e 8 anos de seguimento, as proporções acumuladas de sobrevida ente os homens foram, respectivamente, de $81,0 \%$ e $42,2 \%(\mathrm{EP}=1,45$; Intervalo de Confiança [IC] 95\% [18,54;24,21]), enquanto para as mulheres essas proporções foram de $91,5 \%$ e $83,0 \%$ $(\mathrm{EP}=1,28$; IC 95\% $[6,96 ; 11,99])$. 


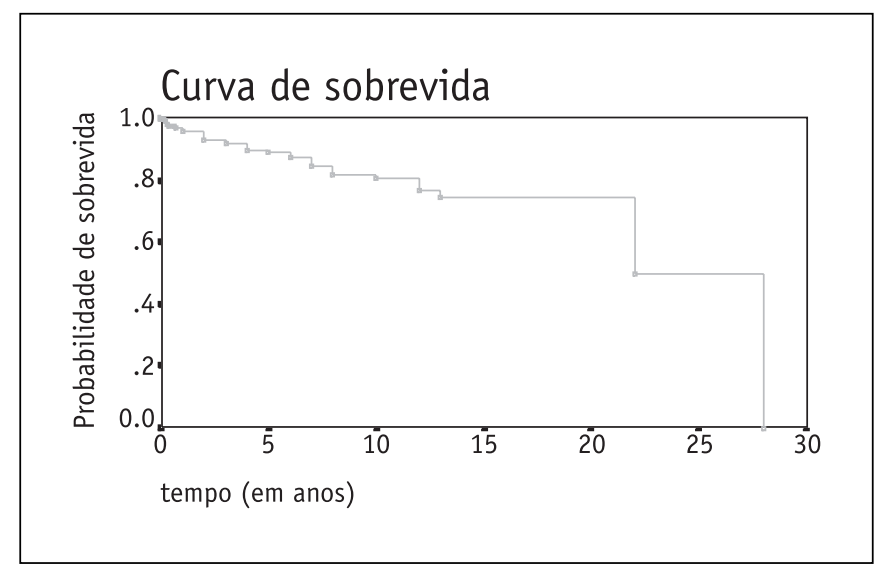

FIGURA 2 - LES: Curva de sobrevida global até o óbito em 509 pacientes.

Com relação à idade dos pacientes, detectou-se diferença estatisticamente significante $(p=0,01)$ entre as curvas de probabilidade de sobrevida acumulada (Figura 3). As proporções acumuladas de sobrevida foram em média de 23,24 anos $(E P=0,76 ;$ IC 95\% $[21,74 ; 24,73])$ para maiores de 16 anos e em média de 14,39 anos (EP=2,0; IC 95\% [10,47; 18,31]) para menores de 16 anos.

Observou-se também diferença estatisticamente significante para sobrevida quanto à ausência de sintomas gerais $(\mathrm{p}=0,02)$ com proporções acumuladas de sobrevida em média de 22,14 anos ( $\mathrm{EP}=1,54$; IC 95\% [19,11; 25,16]) para quem apresentou sintomas gerais ao diagnóstico e em média de 14,37 anos ( $\mathrm{EP}=0,85$; IC 95\% [12,71; 16,03]) para quem não apresentou sintomas gerais ao diagnóstico.

A ausência de artrite ao diagnóstico também estava associada às proporções acumuladas de sobrevida menores $(\mathrm{p}=0,011)$, com média de 13,02 anos $(\mathrm{EP}=0,86$; IC $95 \%$ $[11,33 ; 14,70])$.

A presença de nefrite $(\mathrm{p}=0,009)$ (Figura 4) e a HAS $(\mathrm{p}=0,002)$, tanto ao diagnóstico como durante a evolução estavam associadas às proporções acumuladas de sobrevida menores, com tempo médio de sobrevida, respectivamente, de 12,98 anos ( $\mathrm{EP}=0,88$; IC 95\% $[11,26 ; 14,70])$ e 11,65 anos $(\mathrm{EP}=1,38$; IC 95\% $[8,94 ; 14,36])$.

Ao comparar-se as curvas de probabilidade de sobrevida acumulada segundo o acometimento do sistema nervoso central, verifica-se que elas estatisticamente não diferiram. A presença de convulsão mostrou uma leve tendência $(\mathrm{p}=0,058)$ a um pior prognóstico.

Quanto às demais variáveis clínico-laboratoriais, não foram observadas diferenças estatisticamente significantes nas curvas de sobrevida acumulada.
Quanto à terapêutica utilizada, pacientes que não usaram antimaláricos tiveram curvas de sobrevida acumulada estatisticamente diferentes $(p=0,002)$. O mesmo ocorreu com quem recebeu pulso de metilprednisolona $(p<0,001)$ e ciclofosfamida $(\mathrm{p}=0,002)$.

Pacientes que apresentaram algum tipo de infecção ao longo da evolução, também tiveram curvas de probabilidade de sobrevida piores que pacientes que não tiveram intercorrências infecciosas $(p=0,004)$.

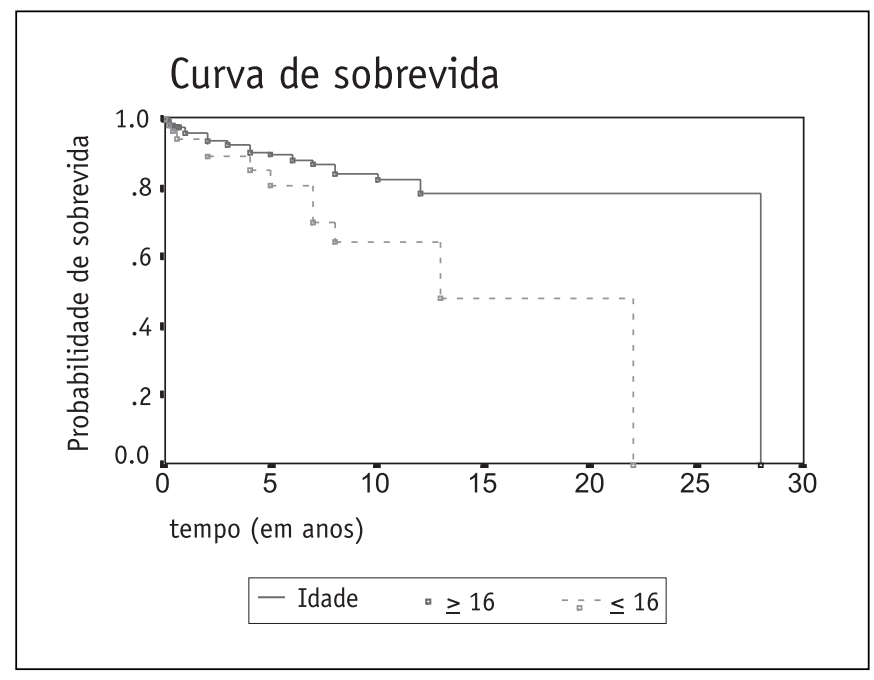

FIgURA 3 - LES: Curva de sobrevida quanto à idade do diagnóstico

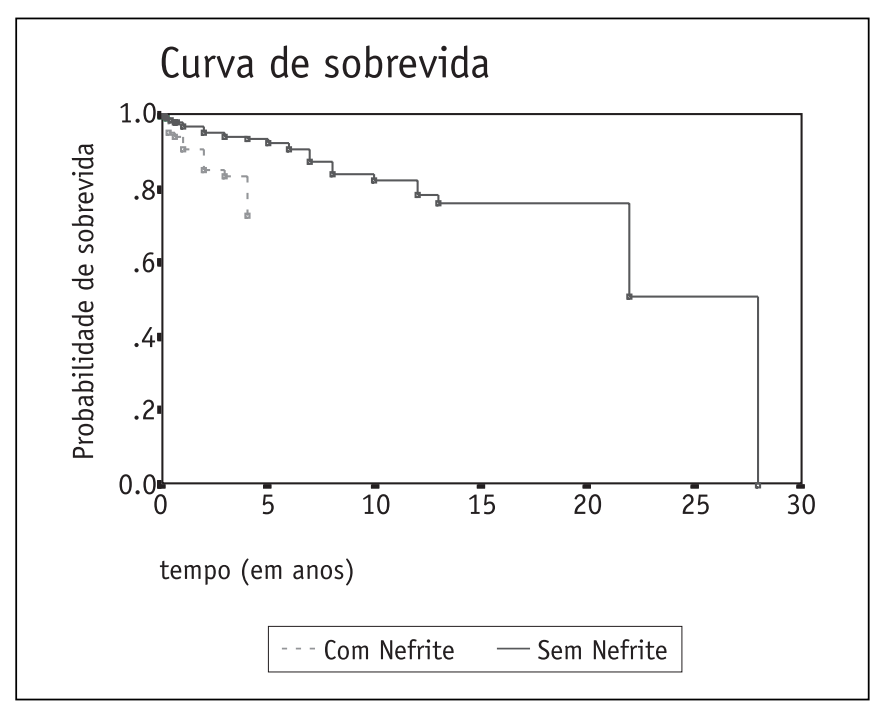

FIgURA 4 - LES: curva de sobrevida quanto a presença de nefrite. 


\section{ANÁLISE UNIVARIADA E MULTIVARIADA}

$\mathrm{Na}$ análise univariada as variáveis associadas ao óbito e as que foram selecionadas para elaboração do modelo multivariado foram a presença ao diagnóstico e durante a evolução de nefrite $(\mathrm{p}=0,0018 ; \mathrm{OR}=2,5 ; \mathrm{IC}=1,4-4,4)$, hipertensão arterial $(\mathrm{p}=0,005 ; \mathrm{OR}=3,2 ; \mathrm{IC}=1,6-6,3)$ e anti-dsDNA $(p=0,032 ; O R=1,8 ; \mathrm{IC}=1,0-3,0)$. A ausência de sintomas gerais ao diagnóstico $(\mathrm{p}=0,025 ; \mathrm{OR}=0,5, \mathrm{IC}=0,3-0,9)$, artrite ao diagnóstico $(p=0,014, \mathrm{OR}=0,5 ; \mathrm{IC}=0,2-0,9)$ e uso de antimaláricos durante a evolução $(p=0,004 ; \mathrm{OR}=0,3$; $\mathrm{IC}=0,2-0,6)$ também estavam associados ao óbito, assim como a presença de infecção durante a evolução $(p=0,005$; $\mathrm{OR}=2,2 ; \mathrm{IC}=1,3-3,7)$ e a idade ao diagnóstico inferior a 16 anos $(\mathrm{p}=0,014 ; \mathrm{OR}=2,2 ; \mathrm{IC}=1,1-4,3)$.

$\mathrm{O}$ modelo multivariado final da análise de sobrevida global pelo modelo de riscos proporcionais de Cox está na Tabela 2 .

Dessa análise, notou-se que para os pacientes com HAS, com infecções durante a evolução, com idade menor de 16 anos e sem artrite o risco de ocorrer o evento óbito foi cerca de duas vezes maior que nos pacientes sem estas características. Já para os pacientes sem uso de antimaláricos o risco de ocorrer o evento óbito foi 3,496 vezes maior que nos pacientes com uso de antimaláricos.

\section{DISCUSSÃO}

Neste estudo, os valores estimados das proporções de sobrevida global foram de $96 \%$ no 1.0 ano, $93 \%$ aos 2 anos, $88 \%$ aos 5 anos, $80 \%$ aos 10 anos e $75 \%$ aos 20 anos de doença, estimativas semelhantes às relatadas em trabalhos recentes $^{(4,5,6,7,8)}$. O aumento nas estimativas das probabilidades de sobrevivência foi notadamente marcante a partir da década de $70^{(16)}$, quando se observou em seguimento por 10 anos de 146 pacientes que as taxas de sobrevida subiram para próximo de $95 \%$ aos 5 anos e $90 \%$ aos 10 anos, melhora esta justificada por métodos clínico-laboratoriais mais padronizados e precisos, favorecendo o diagnóstico mais precoce, bem como maior suporte terapêutico. $\mathrm{Na}$ década de 90, essas porcentagens se mantiveram, aproximadamente, dentro destes valores ${ }^{(4,6,7,8)}$, com exceção do trabalho de Seleznik e Fries ${ }^{(17)}$, que encontraram taxas de sobrevida 64\% aos 10 anos de seguimento.

O pior prognóstico do LES em crianças observado neste estudo tem sido amplamente relatado na literatura ${ }^{(18,19,20)} \mathrm{e}$ principalmente por nosso grupo ${ }^{(21)}$.

Quanto às manifestações clínicas, observou-se uma associação negativa entre sintomas gerais e articulares e a sobre-
TABELA 2

Resultado Final da Regressão de Cox (N=509) AnÁlise MULtivariada

\begin{tabular}{lccc}
\hline Variável & p-valor & Risco & I.C. 95\% \\
\hline Artralgia/artrite & 0,0073 & 0,450 & $0,251-0,806$ \\
Hipertensão & 0,0226 & 2,249 & $1,120-4,515$ \\
Antimaláricos & 0,0001 & 0,286 & $0,154-0,533$ \\
Infecção & 0,0032 & 2,342 & $1,330-4,124$ \\
Idade ao diagnóstico & 0,0040 & 2,611 & $1,359-5,018$ \\
\hline
\end{tabular}

Odds Ratio: razão de risco; IC: intervalo de confiança

Obs.: não foram consideradas os HD Outras-OA, apenas HD 'Não-OA' vs 'OA Mãos'.

vida. Estes resultados não foram relatados previamente, mas poderiam ser explicados pelo pouco conhecimento do LES pela população em geral e o atraso do paciente em procurar o reumatologista quando ausentes os sintomas articulares.

O pior prognóstico dos pacientes com nefrite observados nesta coorte é consensual ${ }^{(2,8)}$, por ser indicativo de uma doença sistêmica mais grave, com necessidade do uso de imunossupressores, predispondo a maior risco de infecção. Entretanto Abu-Shakara et al. ${ }^{(22)}$ concluíram que as curvas de sobrevida dos pacientes com nefrite foram melhores que dos pacientes sem nefrite, justificado pelo fato de que o tratamento da inflamação renal auxiliaria no controle da doença como um todo e, portanto, na melhora do prognóstico.

$\mathrm{O}$ presente estudo demonstrou que pacientes com HAS apresentaram uma curva de probabilidade de sobrevida acumulada pior $(p=0,002)$ que aqueles sem HAS, o que poderia ser justificado pela presença de nefropatia e/ou uso de corticoterapia. Seleznick e Fries ${ }^{(17)}$ verificaram em seu trabalho que a única variável associada com o óbito foi a HAS com um aumento de risco de mortalidade em $2 \%$ para cada milímetro de aumento na pressão arterial sistólica. A HAS não tratada adequadamente é reconhecidamente associada à aterosclerose, às doenças cardiovasculares e aos acidentes vasculares cerebrais ${ }^{(23,24)}$.

Ao comparar-se as curvas de probabilidade de sobrevida acumulada segundo a presença ou não do acometimento do sistema nervoso central, verificou-se que ao contrário do observado por outros autores ${ }^{(8,11)}$, não houve diferença estatística entre os dois grupos. Porém, a presença de convulsão mostrou uma leve tendência $(p=0,058)$ para um pior prognóstico. A ausência de associação poderia ser justificada pela pequena freqüência deste evento neste estudo, ocorrendo somente em 6,80\% dos pacientes.

Muito embora a plaquetopenia tenha sido descrita como um fator de mau prognóstico no $\operatorname{LES}^{(8,22)}$, não foram encon- 
tradas associações entre alterações hematológicas e menor sobrevida nesta coorte.

Com a melhora da sobrevida em decorrência do uso de imunossupressores e antibióticos, as complicações tardias associadas ao tratamento têm aumentado em freqüência, como, por exemplo, a osteoporose induzida por corticoterapia e a aterosclerose. Eventos cardiovasculares foram relatados somente em três pacientes, e não estiveram associados a maior incidência de óbitos. Além disso, a média de idade atual dos pacientes foi de 31,2 anos e com relativo curto tempo de doença e de seguimento. As complicações relacionadas com a aterosclerose geralmente são vistas em uma faixa etária mais avançada e com tempo de doença mais longo, o que poderia justificar a baixa incidência de eventos cardiovasculares nesta coorte.

Trabalhos anteriores mostraram que, de todos os autoanticorpos, o que está mais relacionado com a atividade clínica da doença, bem como com o acometimento renal no LES é o anti-dsDNA ${ }^{(25-27)}$. Embora indiretamente o anti-dsDNA possa associar-se ao óbito pela sua frequiência em pacientes com nefrite, neste estudo o anti-DNA estava associado ao óbito somente na análise inicial, não apresentando força de associação na análise univariada, nem na multivariada.

Ao comparar-se as curvas de probabilidade de sobrevida acumulada segundo a presença de auto-anticorpos não encontramos nenhuma associação. Embora alguns autores ${ }^{(28,29)}$ tenham encontrado doenças mais graves em pacientes com anti-Sm, Gulko et al. ${ }^{(30)}$ não encontraram efeito de risco deste auto-anticorpo para o óbito, o que também não foi observado no presente estudo.

O amplo uso de imunossupressores para o controle da atividade da doença tem diminuído o número de óbitos

\section{REFERÊNCIAS}

1. Carbotte RM, Denburg SD, Denburg JA: Prevalence of cognitive impairment in systemic lupus erythematosus. J Nerv Ment Dis 174: 357-64, 1986.

2. Harvey AM: Systemic Lupus erythematosus: review of literature and clinical analysis of 138 cases. Medicine 33: 291-437, 1954.

3. Merrel M, Shulman E: Determination of prognosis in chronic disease, illustred by lupus erythematosus. J. Chronic Dis 1: 12-32, 1955.

4. Ganczareczyk L, Urowitz MB, Gladman DD: Latent lupus. J Rheumatol 16: 475-8, 1989

5. Abu-Shakra M, Urowitz MB, Gladman DD, Gough J: Mortality studies in systemic lupus erythematosus. Results from a single center. I. Causes of death. J Rheumatol 22: 1259-64, 1995.

6. Gripenberg M, Helve T: Outcome of systemic lupus erythematosus. A study of 66 patients over 7 years with special reference to the secundários à atividade da doença ${ }^{(4)}$, aumentando porém o número de óbitos relacionados com as infecções. As diferenças das curvas de probabilidade de sobrevida acumulada para pacientes que receberam pulso de prednisolona $(\mathrm{p}<0,001)$ e pulso de ciclofosfamida $(\mathrm{p}=0,002)$ para os que não receberam, observadas no presente estudo, poderiam ser explicadas por este fato. Além disso, estes pacientes têm uma doença sistêmica mais ativa, justificando as doses maiores de imunossupressores.

A associação do óbito à ausência do uso de antimaláricos não tem foi relatada previamente. Os antimaláricos são geralmente utilizados para pacientes com manifestações clínicas menos graves, o que poderia justificar porque, neste trabalho, pacientes que não faziam uso de antimaláricos (portanto pacientes com manifestações clínicas mais importantes) tiveram pior sobrevida. Recentemente temse demonstrado o efeito benéfico dos antimaláricos sobre o perfil lipídico de pacientes lúpicos ${ }^{(31)}$, porém, não se tem relacionado diretamente este achado a melhor sobrevida nestes pacientes.

A infecção, freqüentemente associada à imunossupressão, tem sido implicada como a principal causa de óbito no $\operatorname{LES}^{(7,9,12)}$. O mesmo foi verificado no presente estudo, pois pacientes com infecções ao longo da evolução da doença apresentam curvas de probabilidade de sobrevida acumulada piores que pacientes sem intercorrências infecciosas.

A conclusão deste trabalho mostra uma excelente taxa de sobrevida nesta casuística de 509 pacientes com LES. A proporção de pacientes que sobreviveram após 1, 5,10 e 20 anos de seguimento foi de, respectivamente, 96\%, 88\%, $80 \%$ e $75 \%$, comparável às maiores séries que tratam de sobrevida.

predictive value of anti DNA determination. Scand J Rheumatol 20: 104-9, 1991.

7. Pistiner M, Wallace DJ, Nessim S, Metzger AL, Klinenberg JR: Lupus erythematosus in the 1980s: a survey of 570 patients. Semin Arthritis Rheum 21: 55-64, 1991.

8. Reveille JD, Bartolucci A, Alarcon GS: Prognosis in SLE: negative impact of increasing age in onset, black race and thrombocytopenia, as well as causes of death. Arthritis Rheum 33: 37-48, 1990.

9. Gladmann DD: Prognosis of systemic lupus erythematosus and factors that affect it. Curr Opin Rheumatol 4: 681-7, 1992.

10. Sato EI, Vieira AL, Porchini AC, et al: Fatores prognósticos no lúpus eritematoso sistêmico. Rev Bras Reumatol 35: 235-41, 1995.

11. Sibley JT, Olszynski WP, Decoteau WE, Sundaram MB: The incidence and prognosis of central nervous system disease in SLE. J Rheumatol 19: 47-52, 1992. 
12. Hellmann DB: Fatal infection in systemic lupus erythematosus: the role of opportunistic organisms. Medicine 66: 341-8, 1987.

13. Tan EM, Cohen AS, Fries JF, et al: The 1982 revised criteria for the classification of systemic lupus erythematosus. Arthritis Rheum 25: 1271-7, 1982.

14. Kaplan EL, Meier P: Nonparametric estimation from incomplete observations. J AM Sat Assoc 53: 457-81, 1953.

15. Cox DR: Regression models and life tables. J R Stat Soc 34: 187-202, 1972.

16. Fries JF, Weyl S, Holman HR: Estimating prognosis in systemic lupus erythematosus. Am J Med 57: 561-5, 1974.

17. Seleznick MJ, Fries JF: Variables associated with decreased survival in SLE. Semin Arthrits Rheum 21: 73-80, 1991.

18. Kaufman LD, Gomez-Reino JJ, Heinicke MH, Gorevic PD: Male lupus: retrospective analysis of the clinical and laboratory features of 52 patients, with a review of the literature. Semin Arthritis Rheum 18: 189-97, 1989.

19. Schaller J: Lupus in childhood. Clin Rheumatol 8: 219-50, 1982.

20. Tucker LB, Menon S, Schaller JG, Isenberg DA: Adult and childhood-onset SLE: a comparison of onset, clinical features, serology and outcome. Br J Rheumatol 34: 866-72, 1995.

21. Costallat LTL, Coimbra AMV: SLE: clinical and laboratory aspects related to age at disease. Clin Exp Rheumatol 12: 603-7, 1994.

22. Abu-Shakra M, Urowitz MB, Gladman DD, Gough J: Mortality studies in systemic lupus erythematosus. Results from a single center. II. Predictor variables for mortality. J R heumatol 22: 1265-70, 1995.
23. Rothfield NF: Cardiopulmonar manifestations. In: Schur, Phed. The clinical management of SLE. Orlando, Grune \& Startton, 11322, 1983.

24. Wallace DJ, Podell T, Weiner J, Klinenberg JR, Forouzesh S, Dubois EL: Systemic lupus erythematosus--survival patterns. Experience with 609 patients. JAMA 245: 934-8, 1981.

25. Rothfield NF: SLE: clinical aspects and treatment. Arthritis and allied conditions, Philadelphia, L\&F, 1155-77, 1993.

26. Manager K, Manger K, Repp R, et al: Definition of risk factors for death, end stage renal disease, and thromboembolic events in a monocentric cohort of 338 patients with systemic lupus erythematosus. Ann Rheum Dis 61:1065-70, 2002.

27. Spiera RF, Homel P, Spiera H: Long-term outcome in lupus nephritis. Ann Intern Med 137: 545-6, 2002.

28. Beaufils M, Kouki F, Mignon F, Camus JP, Morel-Maroger L, Richet $\mathrm{G}$ : Clinical significance of anti-Sm antibodies in systemic lupus erythematosus. Am J Med 74: 201-12, 1983.

29. Latorre LC: Caracterização tecidual e sorológica de complexos Sm e RNP na nefropatia lúpica, São Paulo, 1987. (Dissertação MestradoFMUSP).

30. Gulko PS, Reveille JD, Koopman WJ, Burgard SL, Bartolucci AA, Alarcon GS: Survival impact of autoantibodies in systemic lupus erythematosus. J Rheumatol 21: 224-8, 1994.

31. Borba EF, Bonfa E: Longterm beneficial effects of chloroquine diphosphate on lipoprotein profile in SLE patients with and without steroid treatment. J Rheumatol 4: 780-5, 2001. 